\title{
DIÂMETRO MÉDIO PREDOMINANTE DE PARTÍCULAS DO SOLO ${ }^{(1)}$
}

\author{
C. L. PREVE DELLO(2), M. S. COSTABILE ${ }^{(3)}$, \\ A. B. STRAPASSON ${ }^{(3)}$, A. P.R. SANTOS ${ }^{(3)} \&$ M. A. AFONSO(3)
}

\begin{abstract}
RESUMO
Apresenta-se um modelo empírico capaz de representar, analiticamente, a curva de distribuição textural acumulada de partículas de um solo e, a partir desse modelo, obter uma expressão que fornece o diâmetro médio predomi nante de partículas $\left(\varnothing_{\mathrm{m}}\right)$, que representa o tamanho médio de partículas que ocorrem com maior freqüência no solo. Essas expressões podem ser aplicadas tanto na física do solo quanto em estudos geológicos, geomorfológi cos e sedimentológi cos.

Termos de indexação: textura, curva textural, distribuição de partículas.

\section{SUMMARY: CHARACTERISTIC MEAN DIAMETER OF SOIL PARTICLES}

An empirical equation to represent the analytical soil particlesize curve is proposed. Based on this equation, a new expression was obtained to calculate the predomi nant mean diameter of soil particles, which represents the mean size of these particles. The equations can be applied, advantageously, in soil physics as well as in geol ogical, geomorphological and sedimentological studies.
\end{abstract}

Index terms: texture, particlesizecurve, particles distribution.

\footnotetext{
(1) Recebido para publicação em agosto de 1999 e aprovado em junho de 2000.

(2) Professor Titular do Departamento de Solos da Universidade Federal do Paraná - UFPR. Rua dos Funcionários 1540, CEP; 80035050 Curitiba (PR). E-mail: cl preve@agrarias.ufpr.br

(3) Graduando de Agronomia da UFPR. E-mail: abs@bbs2.sul.com.br
} 


\section{NTRODUÇÃO}

Ensaios da física do solo, tanto laboratoriais quanto de campo, são dispendiosos e consomem considerável tempo (Klute, 1973; Centurion et al., 1997; F redl und et al., 1998). I sso é particularmente verdadeiro para a obtenção das funções hidráulicas $K(\theta), D(\theta), c(\theta)$ e $\theta(p / \rho g)$ que, em condições de campo, chegam a requerer até quatro ou mais semanas de trabal ho, só para obtenção de dados (Libardi, 1978). A teoria das variáveis regional izadas (geoestatística) tem mostrado, por outro lado, que essas funções podem estar sujeitas a uma extensa variabilidade espacial e temporal (Vieira et al., 1983). Por isso, a nova geração de pesquisas que visam à obtenção das propriedades físicas do solo tem apontado para a busca de métodos alternativos que resultam em rapidez e baixo custo dos ensaios. Extensa lista de recentes trabalhos (Farouki, 1986; Black \& Tice, 1989; Ahuja et al., 1989; Wagner \& Ding, 1994; Fredlund et al., 1995; F redlund et al., 1997) evidencia a rel ação entre a retenção de água eas propriedades físicas dos sol os não saturados, tais como: percolação profunda, transporte de agentes contaminantes no solo, tensão de cisalhamento, capacidade térmica, condutividade térmica, condutividade hidráulica, além das funções hidráulicas já citadas. N os model os mais recentes, os parâmetros físi cos sol icitados para obtenção da curva de retenção são os dados da curva de distribuição textural, a massa específica do solo seco e a massa específica de partículas. Essa é uma das razões que evidenciam a importância da representação analítica da curva de distribuição textural. Um segundo benefício da representação matemática da curva textural reside nos coeficientes dos parâmetros dessas equações, pelos quais essas curvas poderão até ser catal ogadas e classificadas.

O objetivo deste trabal ho foi propor um modelo empírico para expressar, analiticamente, a curva de distribuição textural acumulada de um solo e, a partir desse modelo, obter uma equação capaz de fornecer o diâmetro médio predominante do solo $\left(\varnothing_{\mathrm{m}}\right)$, isto é, o tamanho médio de partículas que ocorrem com maior freqüência no solo.

\section{CONSIDERAÇÕESTEÓRICAS}

A figura 1 mostra o modo usual de apresentar os resultados da análise do tamanho de partículas para um meio poroso qualquer (curva de distribuição textural acumulada). Na ordenada, representa-se a quantidade percentual acumulada de material sól ido e, na abscissa, os diâmetros das partículas correspondentes, em escala logarítmica.

Uma equação empírica potencialmenteútil para representar a curva sigmoidal da figura 1 pode ser escrita como:

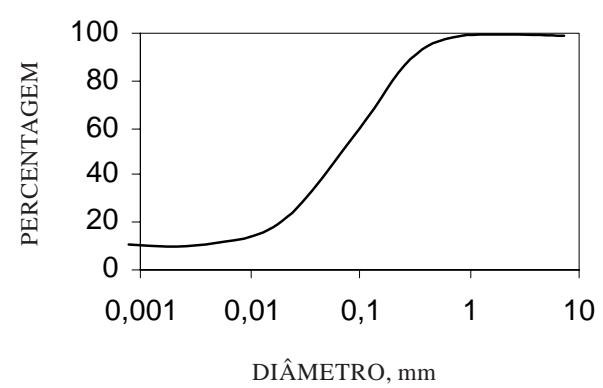

Figura 1. Representação de uma curva de distribuição textural acumulada típica do solo.

$$
P=\gamma+\frac{(100-\gamma)}{\left[1+(\alpha \cdot \phi)^{n}\right]^{1-\frac{1}{n}}}
$$

sendo

$\mathrm{P}=$ percentagem de partículas acumuladas menores que determinado diâmetro (\%);

$\varnothing=$ diâmetro da partícula (mm);

$\gamma=$ parâmetro de ajuste (\%);

$\alpha=$ parâmetro de ajuste $\left(\mathrm{mm}^{-1}\right)$, e

$\mathrm{n}=$ parâmetro de ajuste (adimensional)

Os parâmetros de ajuste da equação (1) podem ser simultaneamente conhecidos pela aplicação da técnica dos mínimos quadrados para ajuste de funções não-lineares (Boratto, 1984).

Quando se relacionam, graficamente, os val ores das tangentes obtidas ao longo da curva textural, considerando o diâmetro das partículas, obtém-se a curva de distribuição de freqüência, a qual passa por um máximo, semel hantemente ao queacontece com a curva de retenção de água no solo (Prevedello, 1996). A representação analítica da curva de distribuição de freqüência, portanto, é obtida derivando-se a equação (1) com relação ao diâmetro $\varnothing$, o que resulta:

$$
\frac{\mathrm{dP}}{\mathrm{d} \phi}=\frac{-\left(1-\frac{1}{\mathrm{n}}\right) \cdot(100-\gamma) \cdot \mathrm{n} \cdot \alpha^{\mathrm{n}} \cdot \phi^{\mathrm{n}-1}}{\left[1+(\alpha \cdot \phi)^{\mathrm{n}}\right]^{2-\frac{1}{\mathrm{n}}}}
$$

O valor de máximo ("pico") pelo qual passa a curva de distribuição de freqüência corresponde, fisicamente, ao diâmetro de partícula que ocorre com maior freqüência no solo. Trata-se de um valor característico da curva de distribuição textural (e, portanto, do solo) eque, por isso, será chamado, neste trabalho, de diâmetro médio predominante de partículas do solo. Analiticamente, o valor de máximo, isto é, o ponto de inflexão na curva de distribuição, é obtido fazendo-se $\frac{\mathrm{d}^{2} \mathrm{P}}{\mathrm{d}^{2}}=0$. Portanto, 
derivando-se mais uma vez a equação (2) e simplificando o resultado, obtém-se

$$
\phi_{\mathrm{m}}=\frac{1}{\alpha}\left[\frac{\mathrm{n}-1}{\mathrm{n}}\right]^{\frac{1}{\mathrm{n}}}
$$

em que $\varnothing_{m}=$ diâmetro médio predominante de partículas do solo $(\mathrm{mm})$.

Tem-se procurado identificar, até por critérios convencionados, o tamanho predominante de partículas que ocorrem com maior freqüência em meios porosos, seja na Física do Solo (Cho et al., 1977), seja na Geologia, Geomorfologia e Sedimentologia (Buffington \& Montgomery, 1999). A capacidade da equação (3) em resumir uma ampla variação de tamanho de partículas sólidas num único valor indica a sua aplicação em estudos geomorfológicos ou hidrossedimentológi cos, em que o tamanho médio predominante dessas partículas poderá ser decisivo na descrição, interpretação e classificação dos fenômenos de transporte.

\section{LITERATURA CITADA}

AHUJ A, L.R.; CASSEL, D.K.; BRUCE, R.R. \& BARNES, B.B. Evaluation of spatial distribution of hydraulic conductivity using effective porosity data. Soil Sci. J ., 148:404-411, 1989.

BLACK, P.B. \& TICE, A.R. Comparison of soil freezing curve and soil water curve data for Windsor sandy loam. Water Res. Res., 25:2205-2210, 1989.

BORATTO, F. BASIC para engenheiros ecientístas. 2. ed. Rio de janeiro, Livros Técnicos e Científicos, 1984. 120p.

BUFFINGTON, J .M. \& MONTGOMERY, D.R. A procedure for classifyng textural facies in gravel-bed rivers. Water Res. Res, 35:1903-1914, 1999.
CENTURION, J .F.; MORAES, M.H. \& DELLA LIBERA, C.L.F. Comparação de métodos para determinação da curva de retenção de água nos solos. R. Bras. Ci. Solo, 21:173-179, 1997.

CHO, T.; NOMURA, T.Y.; SHIKASHO, S. \& INOUE, M. The use of a surface-type and depth neutron moisture meter in studies of soil water regimes. Tottori, Facultty of Agriculture, Tottori University, 1977. 86p.

FAROUKI, O.T. Thermal properties of soils. Clausthal-Zellerfeld, Germany, 1986. p.112-117 (Trans. Technical Publications)

FREDLUND, D.G.; XING, A. \& BARBOUR, S.L. Therelationship of the unsaturated soil shear strength function to the soilwater characteristic curve. Can. Geot. J ., 32:440-448, 1995.

FREDLUND, M.D.; FREDLUND, D.G. \& WILSON, G.W. Prediction of soil-water characteristic curve from grain size distribuition and volumetric-mass properties. In: SIMPÓSI O BRASI LEIRO EM SOLOS NÃO SATURADOS, 3., 1997, Rio deJ aneiro. Anais. Rio dejaneiro, 1997. p.22-25.

FREDLUND, M.D.; WILSON, G.W. \& FREDLUND, D.G. Estimation of hydraulic properties of an unsaturated soil using a knowledge-based system. In: INTERNATIONAL CONFERENCE ON UNSATURATED SOILS, UNSAT'98, Beijing, 1998. p.33-39.

KLUTE, A. Soil water flow theory and its application in field situations. Field soil water regime. Madison, 1973. 212p. (SSSA Special Publication, 5)

LIBARDI, P.L. Condutividade hidráulica do solo em condições de campo. Piracicaba, Escola Superior de Agricultura "Luiz de Queiroz", 1978. 112p. (Tese de Doutorado)

PREVEDELLO, C.L. Física do solo com problemas resolvidos. Curitiba, Salesward-Discovery, 1996. 446p.

VIEIRA, S.R.; HATFIELD, J LL.; BIGGAR, J .W. \& NIELSEN, D.R. Geostatistics theory and applications to variability of some agronomical properties. Hilgardia, 1983, 51:76, 1983.

WAGNER, L.E. \& DING, D. Representing aggregate size distributions as modified lognormal distributions. Am. Soc. Agr. Eng., 37:815-821, 1994. 
C. L. PREVEDELLO et al. 